\title{
Precision alignment and integration of DESI's focal plane using a laser tracker
}

Shourt, William, Besuner, Robert, Silber, Joseph, Dunlop, Patrick, Evatt, Matthew, et al.

William Shourt, Robert Besuner, Joseph Silber, Patrick Dunlop, Matthew Evatt, David Brooks, Peter Doel, Yutong Duan, Kevin Fanning, Enrique Gaztañaga, Paul Martini, Francisco Prada, Michael Schubnell, Gregory Tarle, "Precision alignment and integration of DESI's focal plane using a laser tracker," Proc. SPIE 11445, Ground-based and Airborne Telescopes VIII, 114456J (13 December 2020); doi: 10.1117/12.2562687 


\title{
Precision Alignment and Integration of DESI's Focal Plane using a Laser Tracker
}

\author{
William Shourt ${ }^{*}$, Robert Besuner $^{\mathrm{a}}$, Joseph Silber ${ }^{\mathrm{b}}$, Patrick Dunlop ${ }^{\mathrm{c}}$, Matthew Evatt ${ }^{\mathrm{c}}$, \\ David Brooks $^{\mathrm{d}}$, Peter Doel ${ }^{\mathrm{d}}$, Yutong Duan ${ }^{\mathrm{e}}$, Kevin Fanning ${ }^{\mathrm{f}}$, Enrique Gaztañaga ${ }^{\mathrm{g}}$. Paul Martini ${ }^{\mathrm{h}}$, \\ Francisco Prada, Michael Schubnell ${ }^{\mathrm{f}}$, Gregory Tarle ${ }^{\mathrm{f}}$

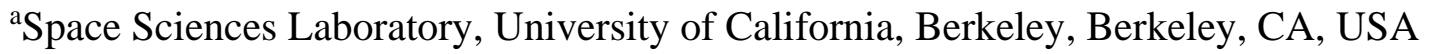 \\ ${ }^{b}$ Lawrence Berkeley National Laboratory, Berkeley, CA, USA \\ 'NSF's National Optical-Infrared Astronomy Research Laboratory, Tucson, AZ, USA \\ ${ }^{\mathrm{d}}$ Department of Physics \& Astronomy, University College London, London, UK \\ 'Physics Dept., Boston University, Boston, MA, USA

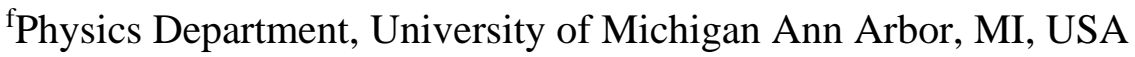 \\ ${ }^{g}$ Institute of Space Sciences (ICE, CSIC), Barcelona, Spain \\ ${ }^{\text {h}}$ Department of Astronomy, The Ohio State University, Columbus, OH, USA \\ iInstituto de Astrofisica de Andalucia CSIC, Granada, Spain
}

\begin{abstract}
The recently commissioned Dark Energy Spectroscopic Instrument (DESI) will measure the expansion history of the Universe using the Baryon Acoustic Oscillation technique. The spectra of 35 million galaxies and quasars over $14000 \mathrm{sq}$ deg will be measured during the life of the experiment. A new prime focus corrector for the KPNO Mayall telescope delivers light to 5000 fiber optic positioners. The fibers in turn feed ten broad-band spectrographs. We describe the use of a Faro Laser Tracker with custom hardware and software tools for alignment during integration of DESI's focal plane. The focal plane is approximately one meter in diameter and consists primarily of ten radially symmetrical focal plane segments ("petals") which were individually installed into the telescope. The nominal clearance between petals is 600 microns, and an alignment accuracy of 100 microns and 0.01 degrees was targeted. Alignment of the petals to their targeted locations on the telescope was accomplished by adjusting a purpose-built alignment structure with 14 degrees of freedom using feedback from the laser tracker, which measured the locations of retroreflectors attached to both the petal and the telescope and whose positions relative to key features were precisely known. These measurements were used to infer the locations of aligning features in both structures, which were in turn used to calculate the adjustments necessary to bring the system into alignment. Once alignment was achieved to within acceptable tolerances, each petal was installed while monitoring building movement due to wind and thermal variations.
\end{abstract}

Keywords: DESI, Dark Energy, Laser Tracker, Retroreflector, Stewart Platform, Hexapod, Alignment, Mayall

\section{INTRODUCTION}

The Dark Energy Spectroscopic Instrument (DESI) was designed to measure the expansion history of the universe with greater precision than previous investigations ${ }^{1}$. It will do so by measuring the spectra of approximately 35 million galaxies and quasars over its five-year survey and employing the Baryon Acoustic Oscillation technique. It can measure up to 5000

*wvshourt@berkeley.edu

Ground-based and Airborne Telescopes VIII, edited by Heather K. Marshall, Jason Spyromilio, Tomonori Usuda, Proc. of SPIE Vol. 11445, 114456J · C 2020 SPIE CCC code: $0277-786 \mathrm{X} / 20 / \$ 21 \cdot$ doi: $10.1117 / 12.2562687$ 
of these spectra at a time by utilizing 5000 fiber positioner robots on its focal plane ${ }^{2}$, each of which can target an individual optical fiber to the focal point of the light from the object being measured. DESI's installation at the Mayall 4-meter telescope at Kitt Peak National Observatory was completed in $2019^{3}$.

The positioners (as well as their supporting hardware) are contained in the DESI Focal Plane Assembly (or FPA). The FPA is broken into multiple constituent assemblies: the Focal Plane Adapter (FPD), the Focal Plane Ring (FPR) and ten Focal Plane Petals (FPP, or 'petals'). The FPD and FPR form the interface between the DESI corrector barrel and the petals (Figure 1). The ten FPP assemblies are radially symmetrical and comprise the actual focal plane. Each petal holds 500 optical fiber positioners, which are mounted in precisely machined threaded holes in a monolithic aluminum wedge (Figure 2). This aluminum wedge also contains alignment features which correspond to their respective features in the FPR. Behind the wedge, a support structure contains all of the electronics which drive the positioners, as well as guides and conduits for the fibers. All of these components are contained with the $36^{\circ}$ segment defined by the aluminum wedge. Each petal terminates in a single cable, approximately 49 meters in length, which contains all 500 optical fibers. The fiber cable terminates in a slithead which will be attached to one of DESI's 10 spectrographs.

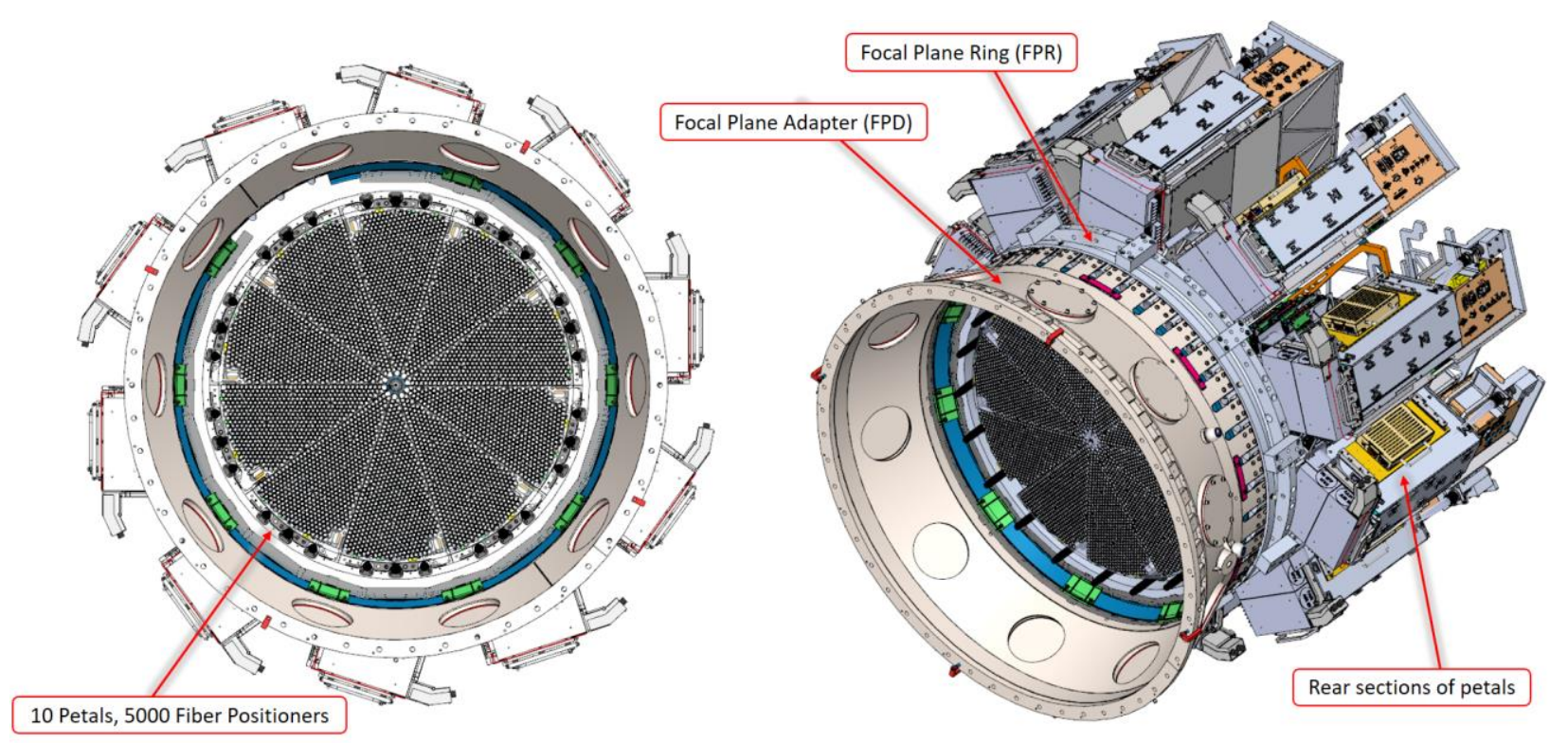

Figure 1: The DESI Focal Plane Assembly (FPA). (Left) A view of the FPA from the perspective of the corrector, showing the ten, radially symmetrical petals and simplified representations of the 5,000 fiber positioners. (Right) A view of the FPA showing the FPD, FPR, and rear sections of the petals. 


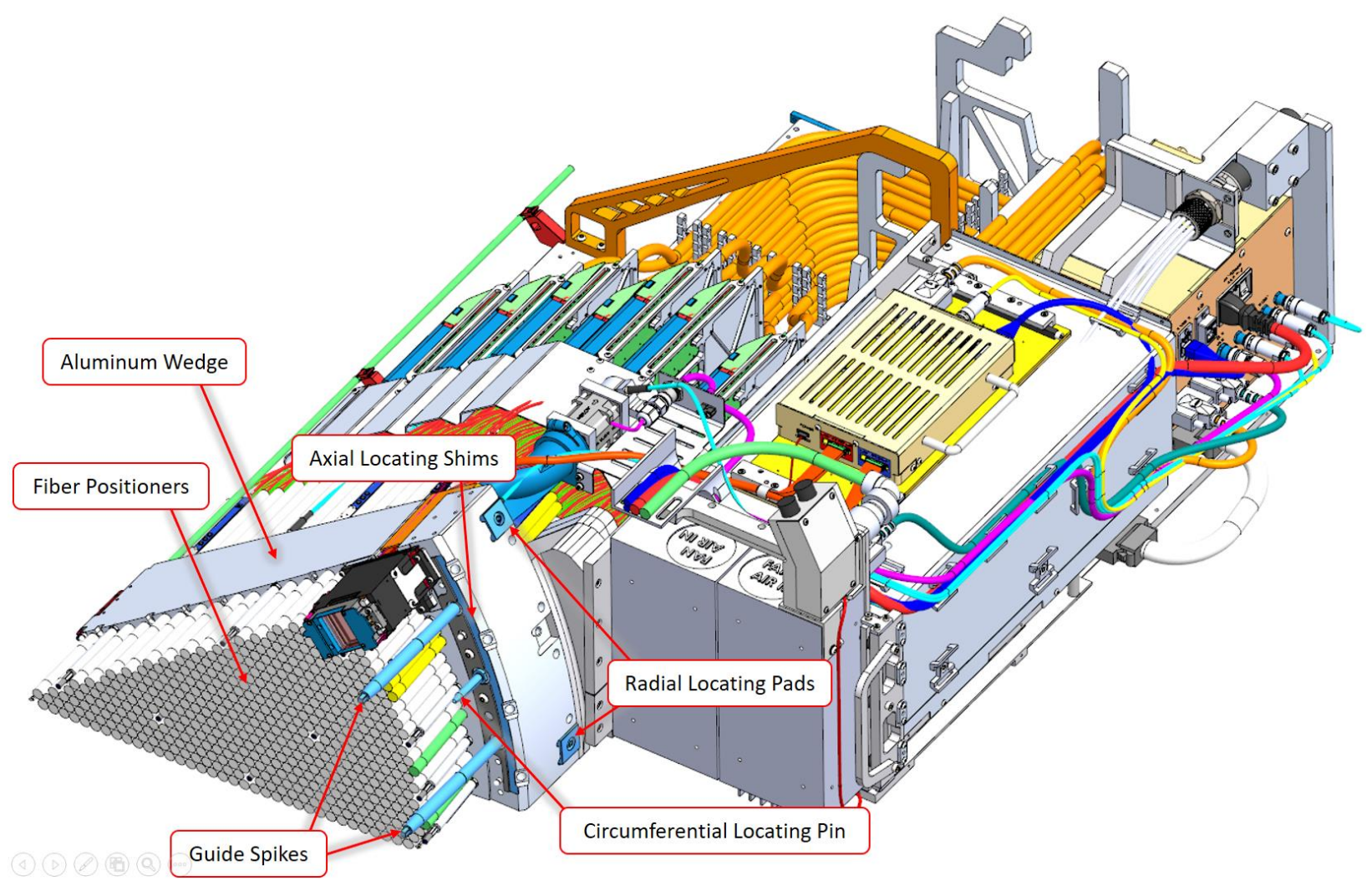

Figure 2: The Focal Plane Petal (FPP, or "petal”), with critical features labeled.

\section{ALIGNMENT FEATURES}

Each petal wedge was pre-aligned to the FPR at Berkeley $\mathrm{Lab}^{4}$. The petal alignment features are a locating pin for circumferential position (which mate into radially-aligned slots in the FPR), two shimmed blocks along the outer radius for radial position, and shims along the mounting surface for axial position. Additionally, there are two looser guide pins which pass through oversized rings (+0.2 millimeters diametrically) in the FPR. The purpose of these loose guides is to both indicate that the petal being installed is well enough aligned in the FPR to prevent collision of the positioners with adjacent petals, and to constrain the motion of the petal during installation. These guides are significantly longer than the petals' locating pins, and were designed to taper from a +0.2 millimeter diametric clearance to a +1.2 millimeter diametric clearance at the point where the precise locating features on the petal would begin to engage with the FPR (Figure 3). This allows said locating features to define the location and not overconstrain the assembly.

Once in position, each petal was fastened into the focal plane using six M8 x 1.25 fasteners. Two of these were oriented radially and normal to the optical axis, pulling the petal into its radial position by registering locating pads against the FPR. The remaining four fasteners were oriented to also point toward the optical axis, but at a $45^{\circ}$ angle instead of being normal to it. These four fasteners pulled the petal into its axial position, registering the locating shims against the FPR. 


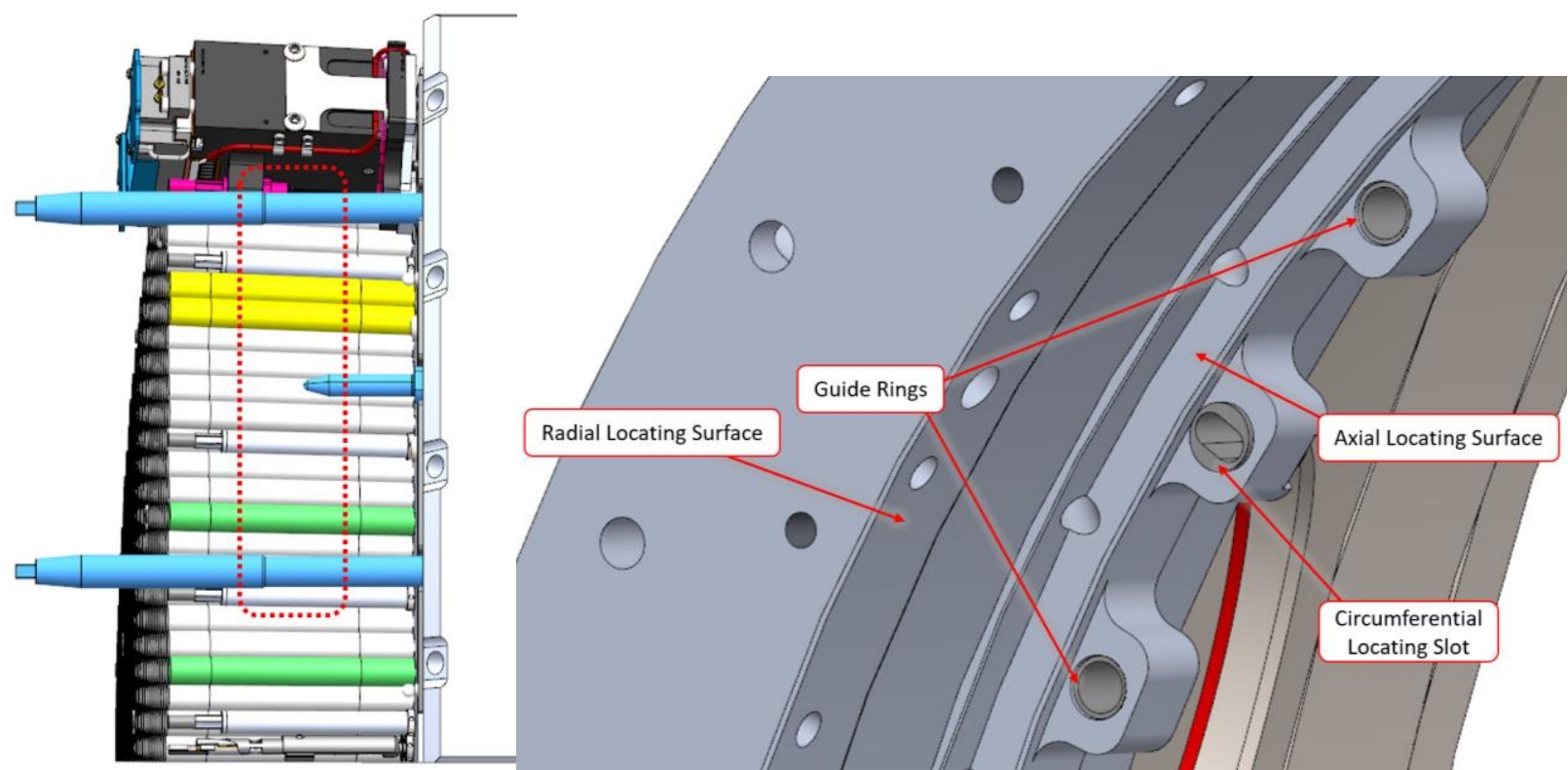

Figure 3: Transition from Guide Spike shoulders to Locating Pin (Left) and FPR locating features (Right)

\section{FOCAL PLANE INTEGRATION REQUIREMENTS}

After much consideration, we decided to integrate the FPA petal-by-petal on the telescope rather than to integrate the FPA off-site and ship the entire assembly. A key consideration was that this plan allowed each of the ten petals to be shipped separately, reducing the risk of sending the entire assembled FPA in a single shipment. Furthermore, it allowed for the post-shipment testing of each petal prior to integration, precluding the possibility of having to remove malfunctioning petals from the FPA prior to its installation on the telescope.

Installing each petal individually on the telescope requires the ability to both precisely align each petal to its corresponding position in the focal plane and carefully insert it into position while avoiding collision with adjacent petals. We performed the installation while the telescope was locked into position at the Southeast Annex, a pre-existing service location with a platform well suited to support our installation. While pointing at the southeast platform, the telescope sits with its optical axis approximately $7.2^{\circ}$ above horizontal, which defines the insertion path. With the nominal separation between petals being 0.600 millimeters and each petal's length being over one meter, this required careful positioning and alignment of both the petal being installed and the axis of insertion into the focal plane. Furthermore, we anticipated significant variations in the strain deformations of the installation hardware between different petal installation locations, as the roughly 60 kilogram petals would be held at very different positions and orientations. As such the angle of the petal would have to be adjustable independent of the angle of installation.

One last complication of note is the fiber cables; each petal has an approximately 49 meter, 25 millimeter diameter fiber cable permanently attached to one end, which required constant management to prevent damage to the optical fibers and interference with integration. 


\section{FOCAL PLANE INTEGRATION HARDWARE}

\subsection{The Standoff Support Leg}

In order to securely hold the petal and precisely locate its alignment features, it was desirable to hold the petal by the aluminum wedge on which those features were located. However, the need to clear any adjacent petals during installation required any mounting for the petal to approach from the end opposite the fiber positioners ("rear"), going deep through the supporting structure to reach the wedge towards the front of the petal. As such, three M6 threaded holes were placed into the wedge in a position accessible from the rear of the petal.

The Standoff Support Leg (or "leg") is a steel weldment designed to reach these fastening points as well as provide multiple points from which a petal could be lifted (Figure 4). It was designed to be used only when moving or installing the petals, and removed after installation. To enable both fastening to the M6 mounting holes and easy tool access while keeping clear of the delicate internals of the petal, three captive fastener rods of over 900 millimeters in length were added to the assembly. A guide rail was added to the leg, as well as a corresponding carriage inside the petal, to both support the rear of the petal during lifting and guide the leg during installation in the petal.

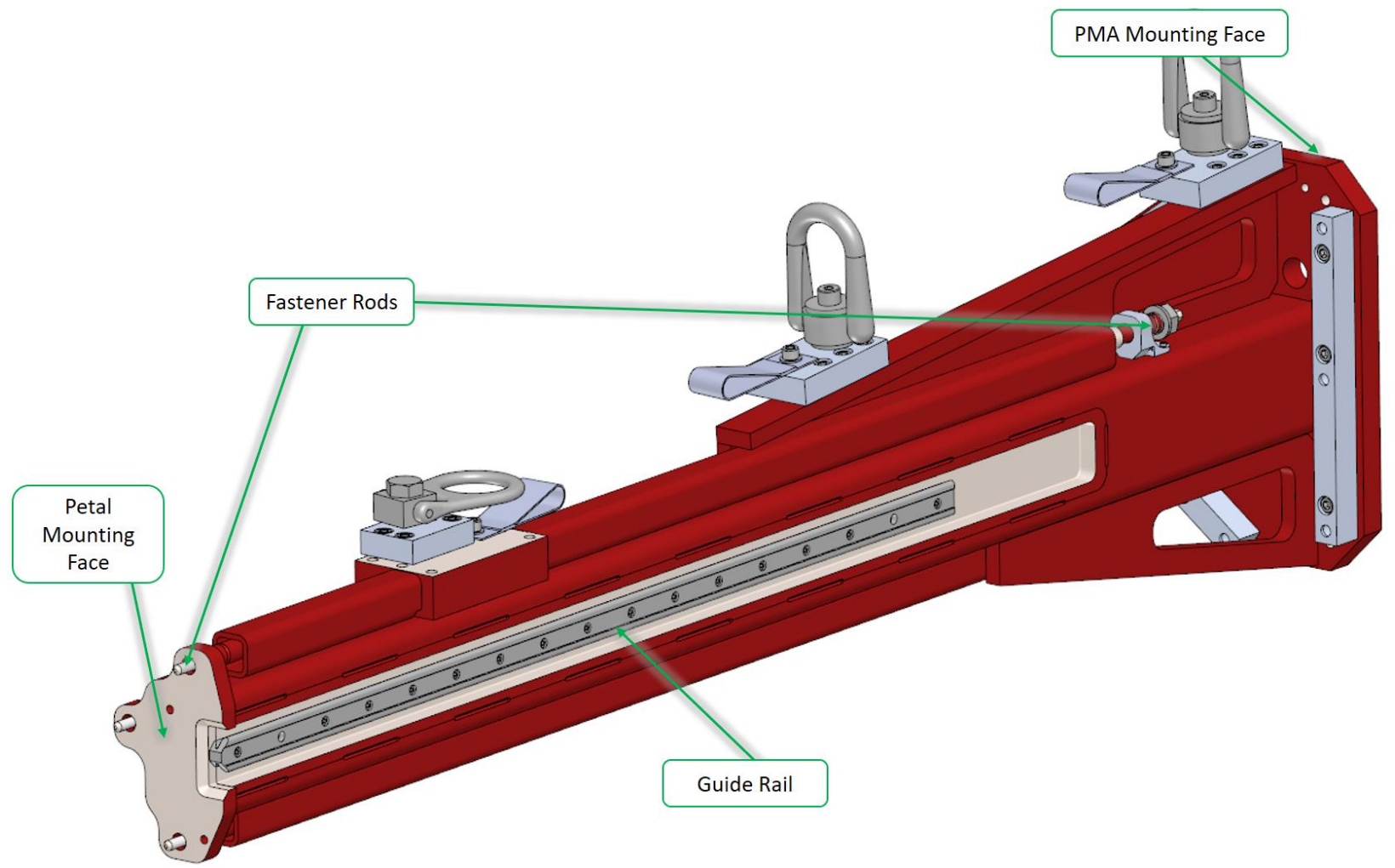

Figure 4: The Standoff Support Leg, with key features noted. Two of the three fastener rods are mostly occluded in this view, but are the same length as the one which is visible.

\subsection{The Insertion Sled}

Proper alignment and installation of the petals required 5 degrees of freedom: 2 axes of translation in the plane perpendicular to the optical axis for positioning, 2 axis of rotation normal to the optical axis for orientation, and 1 axis of translation parallel to the optical axis for petal insertion. The positioning and alignment axes required fine adjustability and relatively short travel. Each petal would need to be lowered into its initial, pre-installation position on the integration hardware using a crane, and then inserted into the focal plane along an axis parallel to the telescope's optical axis. As each 
petal was approximately 1 meter in length, a total travel of approximately 2 meters was desirable to allow clearance for maneuvering of the petals into place without interfering with petals which had already been installed in the focal plane.

These requirements were met by the DESI Insertion Sled (or "sled"), a long linear stage on top of a 6 degree of freedom Stewart platform (Figure 5). This resulted in extra degrees of freedom (a finely adjustable translational axis parallel to the insertion axis as well as a "roll" axis parallel to the insertion axis), but took advantage of a robust and well-defined method of defining position and orientation. Unlike the more common "hexapod" design, this Stewart platform was designed with the adjustable struts oriented along axes that were designed for intuitive translational adjustment. Three struts were oriented vertically (normal to the floor), two aligned parallel to the optical axis, and one parallel to the floor and perpendicular to the optical axis. The locations of all six struts are shown in Figures 5 and 10. Each strut was a turnbuckle-type assembly secured on each end by ball joint end rods (Figure 6). They were designed with right handed differential threads of 8 threads per inch and 12 threads per inch, resulting in an effective pitch of about 0.042 inches or 1.06 millimeters, allowing for fine adjustment in both location and orientation. Their adjustment range was \pm 0.63 inches (16 millimeters) from their nominal length, giving the sled a maximum translational adjustment range of \pm 0.63 inches in each axis, and a rotational range of about $\pm 0.63^{\circ}$ to $\pm 1.25^{\circ}$ depending on the axis of rotation. As the rotation and translation adjustments of a Stewart platform are coupled, these extremes could not be achieved simultaneously. The struts were designed to be manually adjusted by turning their bodies, which were given a hex profile to enable easy use of tools.

The linear stage consisted of a carriage which rode on linear bearings across two parallel linear guide rails. It was driven by a leadscrew, which was in turn driven by hand wheels through a right-angle gearbox. This arrangement was desirable because it allowed for precise and direct control over the petal's position along the insertion axis.

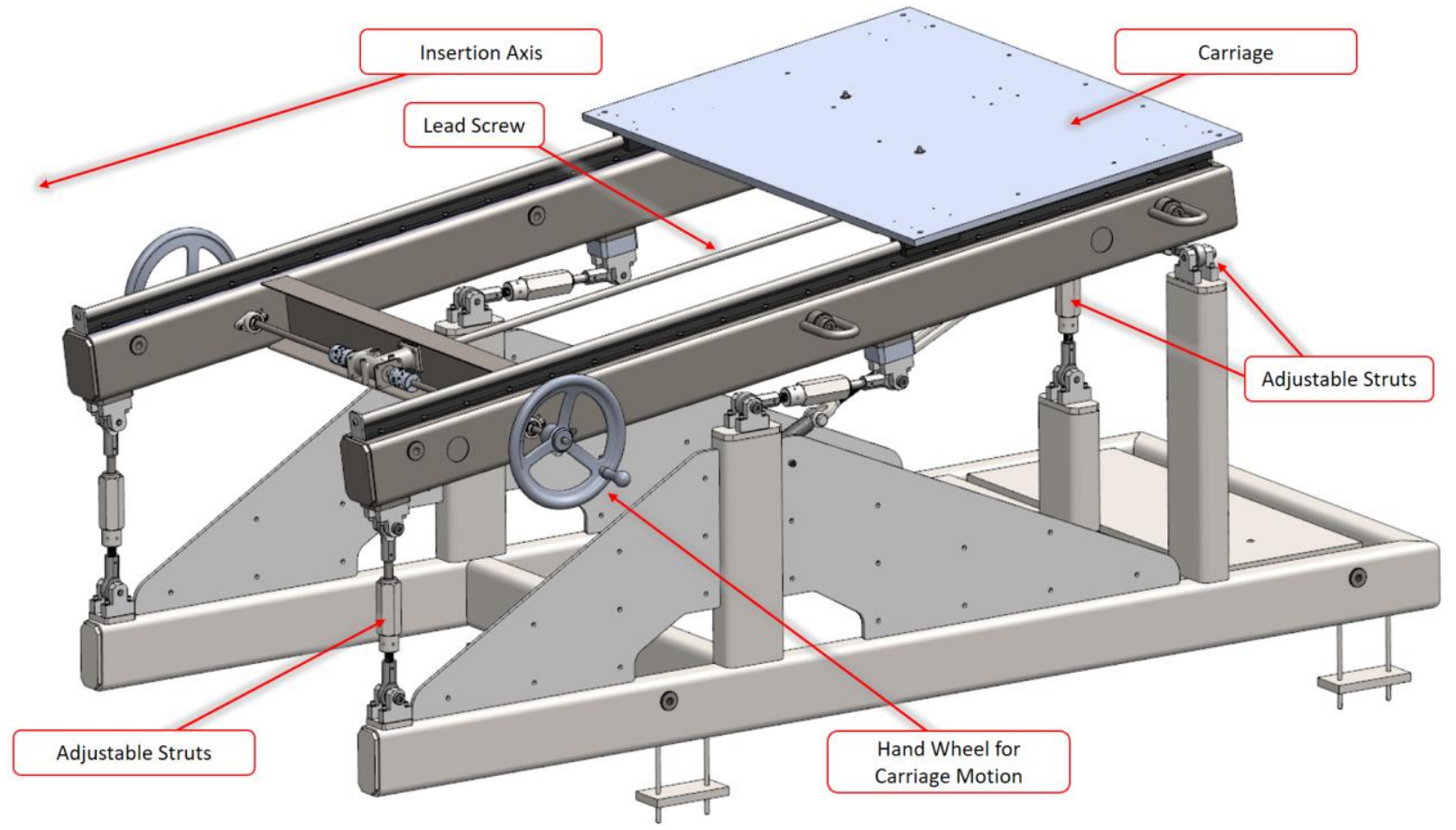

Figure 5: The Petal Installation Sled, with six adjustable struts shown (struts partially occluded here are also shown in Figure 10) 


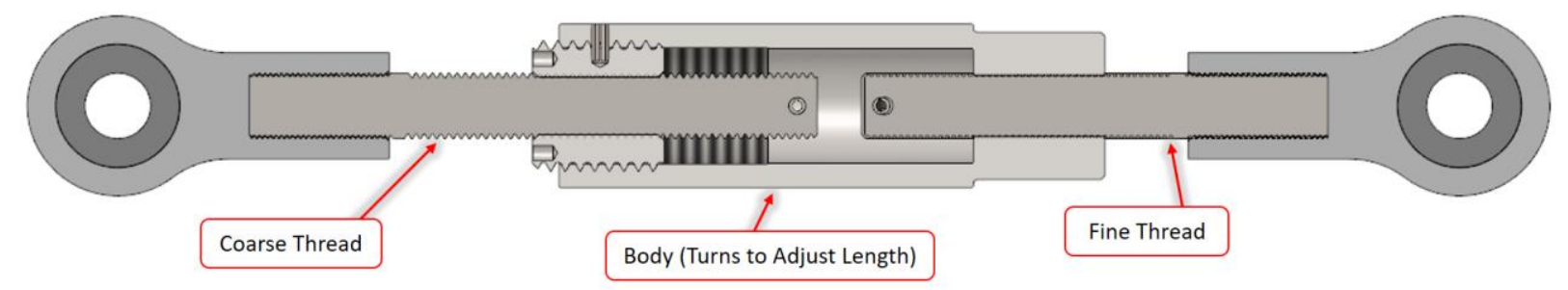

Figure 6: Section View of Sled Adjustable Strut

\subsection{The Petal Mount Adapter}

The sled allows alignment of the insertion axis with the optical axis and translation along said axis. The petals are mounted to the sled with the Petal Mount Adapter (PMA). A key requirement of the PMA was that it must be able to rotate each petal into its respective position on the focal plane. Also, due to difficult to predict structural deformations in the variety of orientations required for the petals, 5 additional degrees of adjustment ( 2 translational and 3 rotational) were required to position and orient the petal independently of the sled.

The PMA was designed to meet these requirements by placing a large rotational stage on top of a second Stewart platform, similar to that of the sled below it (Figure 8). The rotation stage allows the clocking of each petal to the rough position and orientation of its target location in the focal plane, while the Stewart platform allows for the fine tuning of the petal's position and orientation prior to insertion. This again results in an extra degree of freedom (a translational axis parallel to the insertion axis). The rotation stage is driven by a hand wheel through an 80:1 reduction right-angle gearbox. In order to reduce the amount of force required to turn the wheel, a counterweight to balance the petal's mass was added to the side of the rotation stage opposite the petal.

The struts of this Stewart platform are similar to those of the sled, though smaller and more finely adjustable, with an effective pitch of 0.016 inches or 0.40 millimeters per turn. Their adjustment range was \pm 0.25 inches $(6.3$ millimeters) from their nominal length, giving the PMA a maximum translational adjustment range of \pm 0.25 inches in each axis, and a rotational range of about $\pm 0.53^{\circ}$ to $\pm 0.68^{\circ}$ depending on the axis of rotation. As with the sled, these extremes could not be achieved simultaneously.

The PMA struts were designed with inline load cells (Figure 7). The readings of all six of these load cells were used to determine the net forces on the structure. When the petal was mounted on the PMA and hanging freely, the net forces on the load cells would be the combined weight of the petal and PMA. Any additional forces applied to the petal would be visible through the load cells, allowing the installers to determine when the petal began to engage the focal plane, or if it was coming into contact with anything else during its travel along the insertion axis. Additionally, baseline readings for these load cells were taken in every installation position when the PMA was only supporting the Standoff Support Leg, providing a reference for removing the leg after petal installation.

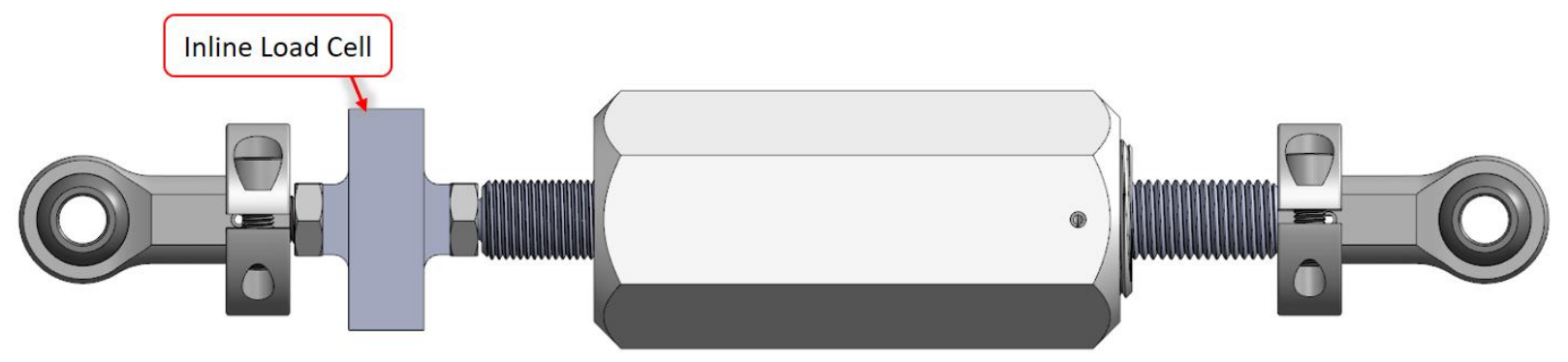

Figure 7: PMA Adjustable Strut 

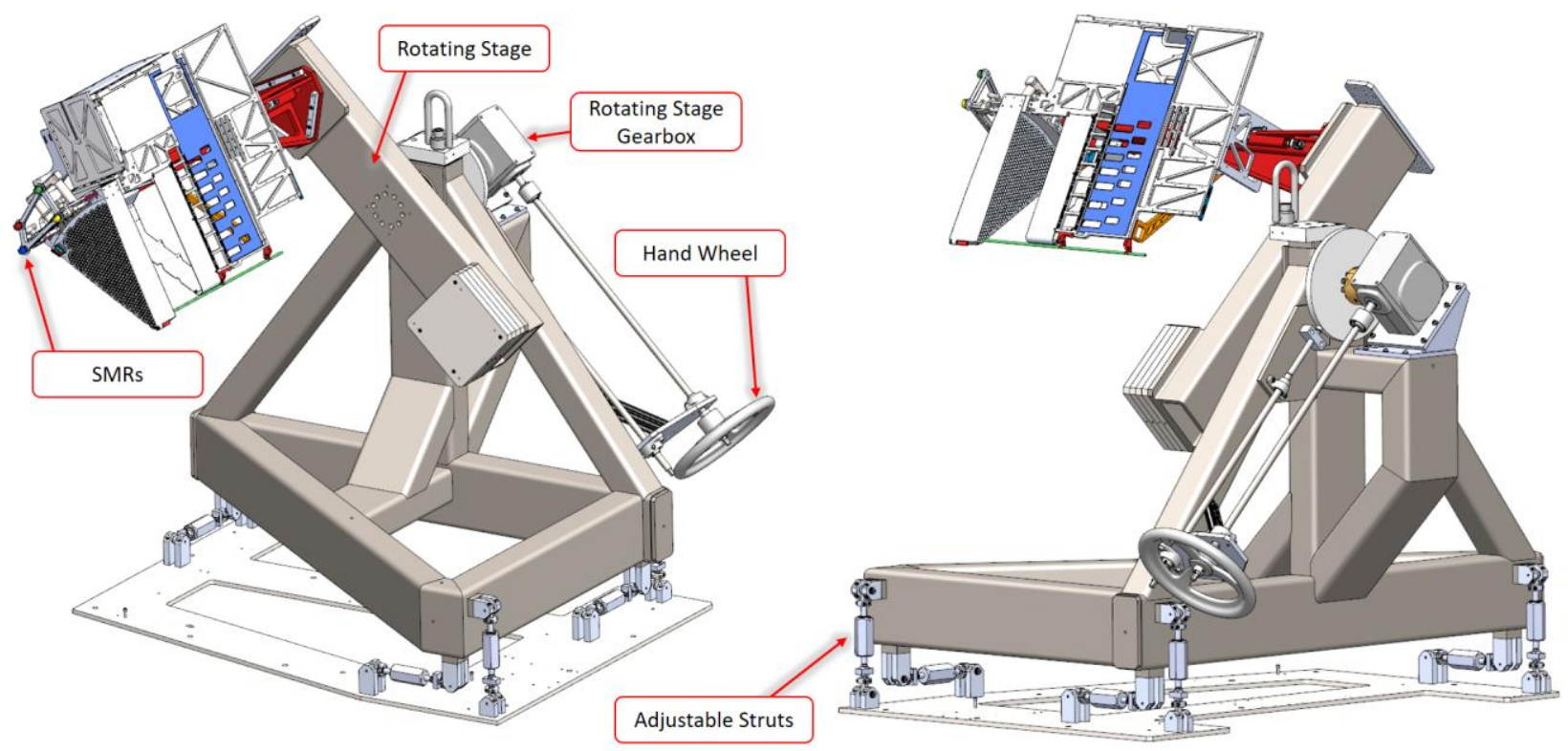

Figure 8: Petal Mount Adapter (PMA) with mounted Petal

\section{ALIGNMENT BETWEEN PETALS AND FOCAL PLANE}

\subsection{Measurement Hardware}

With all of the necessary degrees of freedom made available, we determined that the best method to measure the initial alignment was to use Spherical Mount Retroreflectors (SMRs) mounted to both the FPD on the telescope and the petals, and measured with a Faro VantageE Laser Tracker (Figure 9). The laser tracker is able to measure positions of retroreflectors with a great deal of accuracy. While in the field, uncertainties of under $\pm 100 \mu \mathrm{m}$ were typical, and were under $\pm 50 \mu \mathrm{m}$ under good conditions.

The SMRs are mounted in "nests," which are small, cylindrical pucks which hold the SMRs against 3 repeatable points of contact with magnets. For the measurements of the SMRs to be useful, their relationship to the critical locating features on the petals and FPR must be known. In the case of the FPD/FPR, the nests were bonded to the FPD cone in locations that were designed to be visible during installation. After the nests were bonded, their positions relative to the locating features on the FPR were measured at Berkeley Lab, allowing the positions of said features to be calculated from the measured positions of the SMRs.

Bonding the nests to the petals was not practical, due to both a lack of suitable surfaces and the variety of orientations in which the petals were to be installed. As such, the Laser Target Mount (LTM) was designed to hold an array of SMRs and be repeatably attached and secured to the guide spikes of the petals. This allowed the SMRs and nests to be removed from the petal, while still providing a well-defined relationship between the SMRs and the petal's locating features. 

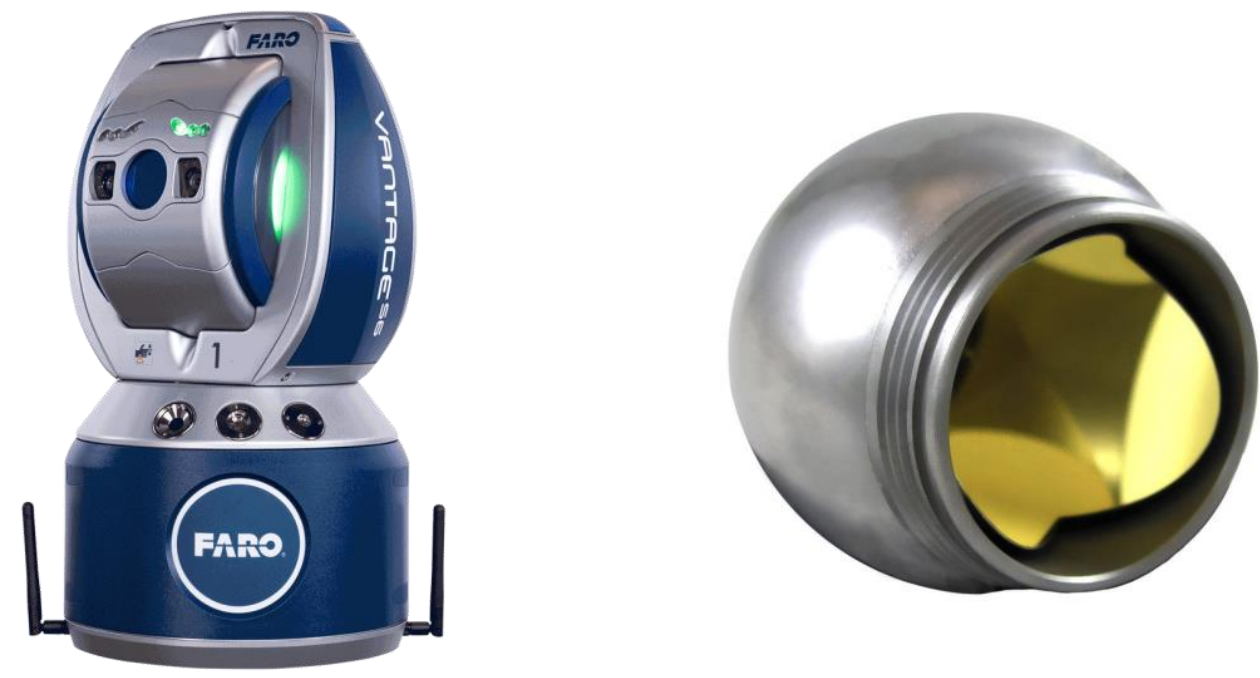

Figure 9: (Left) Faro VantageE Laser Tracker and (Right) Spherical Mount Retroreflector

\subsection{Aligning the Insertion Axis with the Optical Axis}

With the FPD and FPR installed on the telescope, SMRs placed in their nests on the FPD, the telescope secured in the servicing position at the southeast platform, and the PMA/Sled assembly in place on said platform, the first step for installation was to align the insertion axis (the axis along which the sled's carriage travels) to the optical axis of the telescope. Ideally the sled carriage's axis of motion would be parallel to the optical axis, and the center of the PMA's rotation stage coincident with the optical axis. All equipment would be placed as close as possible to its nominal location, and the struts were set to their nominal lengths (center of their range of travel).

The five SMRs on the FPD were used to establish the coordinate system. A measurement was taken of each, then those five points are best fit to their previously measured locations on the FPD. In order to measure the center of rotation of the PMA's rotation stage, an SMR nest is temporarily attached to the rotation stage using hot melt adhesive. The sled carriage is then moved to the rearmost point in its range of motion (furthest from the telescope mirror). An SMR is placed in said nest and the stage is rotated. The laser tracker is used to measure the position of that SMR at a series of points along the entire range of motion. The sled carriage is then moved to its forwardmost position, and the process is repeated to generate a second set of points. Both sets of points (rear and forward) are best fit to circles, the centers of which define the initial insertion axis. We then generated a transformation matrix based on the motions required to make the insertion axis parallel to the optical axis, and to make the forward circle centroid coincident with the optical axis.

At this point it was necessary to calculate the adjustments in strut length that would be required to move the upper stage into alignment. Early in the development of the sled, we determined that attempting to do so by setting exact strut lengths would be unnecessarily complicated and expensive. It would have required the assembly to be constructed with a very high level of precision; a daunting task with large weldments. Additionally, it would have required significant effort to include all of the structural deformations caused by various loadings of the Stewart platforms (and all other structures along the load path) in the calculations. It was decided that a much simpler solution would be to approximate the required changes in length rather than attempt to calculate and measure the absolute strut lengths. We did this by making an inverse kinematic model of the Stewart platform, using the assumption that all struts started at their nominal length and applying the transformation matrix between the measured and targeted orientations to the upper stage of this hypothetical, nominal model. As the cosine errors caused by the deviations of the strut lengths and angles from nominal were quite small, it was 
possible to reach acceptable alignment with a few iterations of this method. This method was used for adjustment to both the sled and PMA Stewart platforms.

The nominal model consisted of a set of 12 points defined by the rotation centers of both ball ends of each of the 6 struts in their nominal positions in the same coordinate system which was used to measure the SMR locations. We applied the previously generated transformation matrix to the set of points which define the strut connections to the top (mobile) part of the sled, while the points defined by the strut connections to the stationary base remain constant. We calculated the Euclidean norms of the mobile, upper stage points relative to their respective static, lower stage counterparts to determine the final strut lengths, which we then subtracted from the nominal strut lengths to determine the required changes in strut length to move the sled to the position prescribed by the transformation matrix.

At this point the strut lengths are adjusted while the length changes are monitored. When the final lengths have been reached, the process of measuring the insertion axis is repeated to determine if the alignment move was successful. As the moves were estimates generated using the (almost certainly incorrect) assumption that all components were perfectly located in their nominal positions (indeed, if they had been no movement would be necessary), the first move will usually bring the system closer to alignment, but will require subsequent adjustments to reach an acceptable alignment. Typically, the alignment will fall within acceptable parameters within 3 to 5 iterations.

\subsection{Aligning the Petal with the FPR}

After the insertion axis alignment, the sled carriage is returned to its rearmost position and a petal is mounted to the PMA (Figure 11). After the petal is secure, the LTM and 4 SMRs are attached. The petal is then adjusted to its rough location in the FPA using the PMA rotation stage and a digital level. This method of rotation was quite coarse when compared to the finer adjustments allowed by the struts, and would sometimes require small adjustments after initial positioning. Once the petal is in its initial position, the locations of the SMRs are measured using the laser tracker. A transformation matrix is then calculated by fitting these points to their calculated optimal positions for petal alignment. As with the sled, this transformation matrix is then applied to the mobile, upper stage coordinates of the strut attachment points of the PMA. For this transformation, the tip of one of the petal's guide spikes was chosen as the center of rotation. Again, we calculated and subtracted the Euclidean norms of the mobile points with respect to their static counterparts on the PMA's base from the nominal strut lengths to provide the approximate adjustments required by each strut to achieve alignment. Additionally, we calculated a rotation adjustment about the optical axis, in case the initial setting of the PMA's coarse rotation stage deviated significantly from the desired position and orientation.

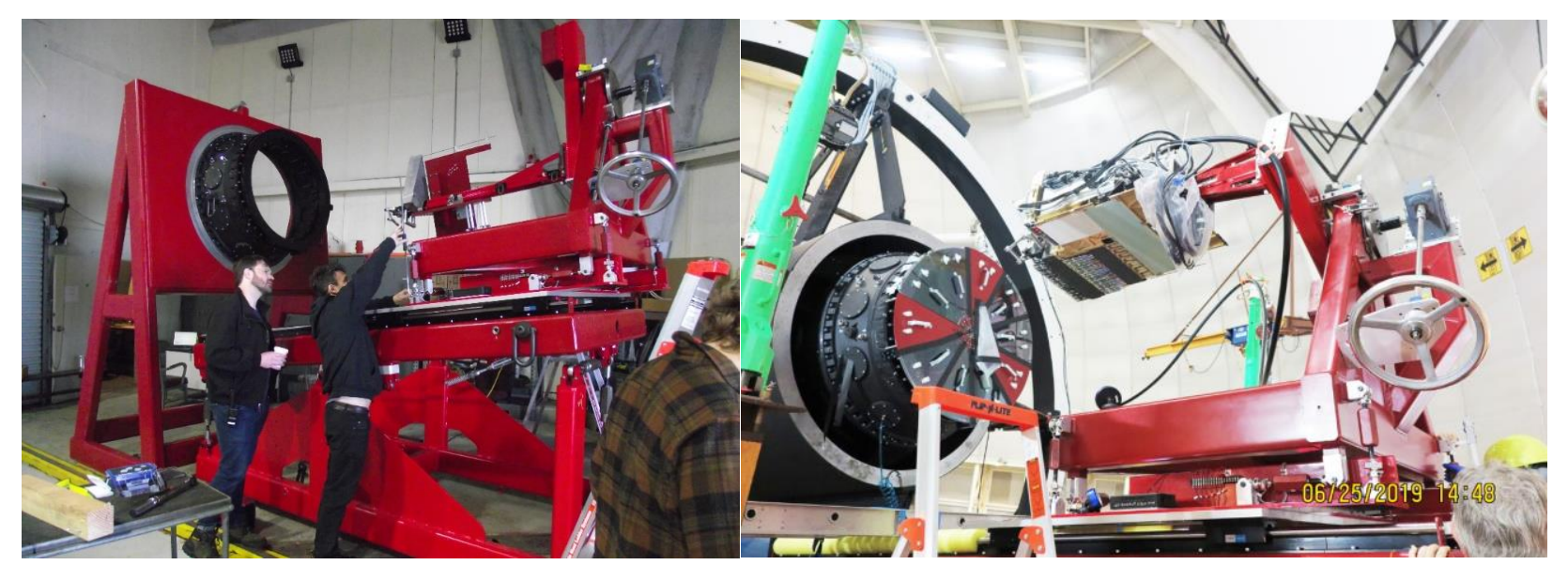

Figure 10: (Left) The authors adjusting the alignment of the PMA in a test installation setup. A dummy petal with similar mechanical features and properties to a production petal is mounted to the PMA. (Right) The first production petal ready for alignment and installation. 


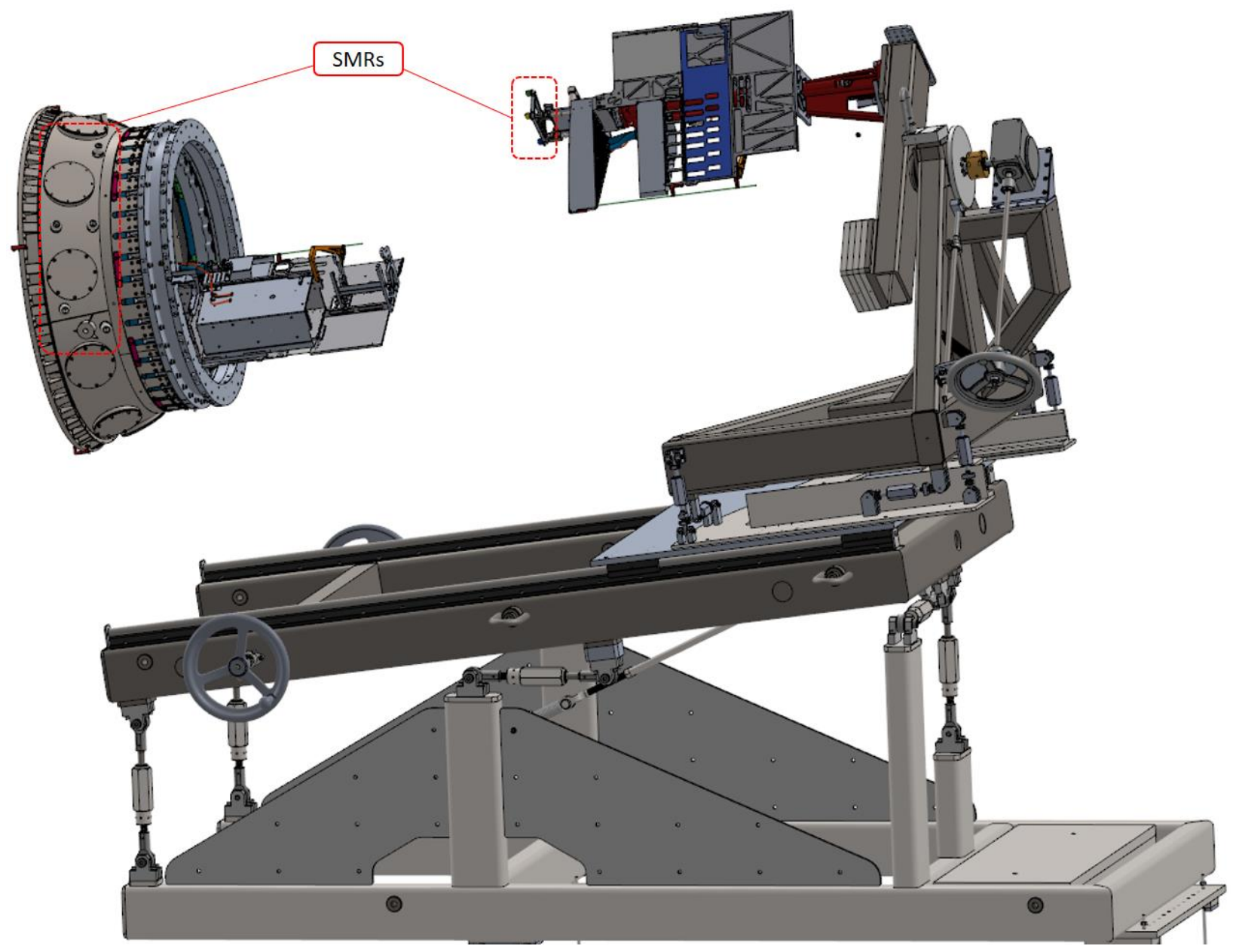

Figure 11: Full Sled/PMA assembly with Petal mounted, and FPD/FPR (telescope not shown)

If the required motion exceeded the \pm 6.4 millimeter range of any of the struts, the rotation stage was adjusted according to the calculated motion. Another measurement of the SMRs would then be taken, and the process would be repeated until the rotation stage was adjusted to within acceptable parameters and the struts could be used for fine adjustment. This process was only necessary occasionally, and was generally resolved with a single adjustment of the rotation stage. Once the rotation stage was in its final position, the struts would be adjusted according to the nominal model calculations. As with the sled adjustments, this generally resulted in an acceptable alignment within 3 to 5 iterations.

Once alignment had been confirmed by measuring the SMR positions, the SMRs and LTM were removed from the petal. At this time the readings of all of the PMA struts' load cells were taken, as a baseline measurement of the forces they experience while fully supporting the petal in its aligned position.

\subsection{Petal Insertion}

After alignment, the petal was inserted into the FPA by driving the sled carriage forward via a hand wheel. This process was carefully monitored visually from multiple angles, as well as instrumentally through the load cell readings. While it was likely possible to calculate the rough location of any applied forces using the load cell readings and their previously established baseline values, the nearly rectilinear arrangement of the struts made reading the load cells quite intuitive. An uneventful insertion would consist primarily of the load cell values remaining relatively static until the weight of the petal 
started to be supported by the FPA during engagement, at which time there may be slight changes in the three "vertical" struts and the one forward "transverse" strut, which were all normal to the axis of insertion. When the axial locating shims met the FPR, the readings of the two struts parallel to the insertion axis would rapidly move from compression towards tension. At this time all sled carriage motion would be stopped, and the alignment of the holes for the mounting fasteners would be confirmed with a borescope. If they were not properly aligned, the sled carriage position would be adjusted until the fasteners could be successfully installed.

Once the petal had been secured to the FPA, the struts were adjusted until their load cells roughly matched the reference values for supporting only the Standoff Support Leg in the current installation position. This was done to relieve any strain in the structure that might cause the Standoff Support Leg to rapidly change position when no longer supporting the weight of the petal. Once the load cells were within roughly $10 \%$ of their reference values, the three fasteners holding the leg to the petal were disengaged. When all fasteners were confirmed to be free of the petal, the sled carriage would slowly be moved away from the FPA to retract the leg from the petal. The PMA strut load cells would be monitored during this process to detect any unexpected forces as the leg was retracted along its guide rail. After being fully retracted, the leg was removed from the PMA, completing the petal installation process.

\subsection{Installation Challenges}

We encountered a number of difficulties during the FPA integration process. Wind was a predictable concern; as one can imagine, a building with a large cross-section situated on a mountaintop can find itself subject to significant wind forces (this author once had his glasses blown off his face by the wind while standing beside said building). These forces lead to significant vibrations throughout the structure, and made careful alignment of the petals to the FPA too risky to justify, if not impossible. While we attempted to quantify a wind level that would preclude installation efforts, in practice our requirements couldn't tolerate much more than a light breeze. This required us to schedule installation days around favorable weather forecasts, and to delay work if the predictions proved inaccurate. Fortunately, the weather during our installation window provided ample opportunity to complete our work.

One complication that we did not foresee was the effect of the varying angles of the sun relative to the building throughout the day. During early installation work, we noticed that the alignment could change significantly over time (up to about 0.030 " or 0.75 millimeters per hour at the extremes). At this point it should be noted that the telescope itself is structurally isolated from the building surrounding it, with both structures emerging separately from the foundation. This arrangement is very helpful in isolating the telescope from vibrations encountered by the building, but by its nature means that the building can move independently of the telescope. The petal installation hardware was mounted to the building, which the FPA (to which we were aligning) was mounted on the telescope. We noticed that the changes in alignment over time matched very closely with what one would expect if the building was expanding on one side and contracting on the other, and tracked very well with the angle of the sun. This led us to surmise that the origin of the issue was the gradual thermal expansion and contraction caused by the varying exposure of the building to sunlight during the course of the day, while the telescope itself remained relatively static. Attempts had been made to secure the telescope to the building's upper floor using a beam bolted at both ends, but this only coupled the two structures to a limited extent and could not prevent this relative motion. As a consequence of this effect, we had to make efforts to rapidly install the petals after the alignment of each had been finalized, before the motion of the building would invalidate the alignment.

\section{SUMMARY}

Testing of the installation equipment began in April of 2019, and after multiple successful test trials, installation of the petals into the FPA was conducted in June and July of 2019. While initial installations took up to two days, towards the end of the installation process we were able to install two petals per day under good conditions. The use of sequential Stewart platforms for alignment proved to be a robust system when combined with precision measuring equipment. 
We were able to complete integration in less time than was anticipated thanks to the skilled work and contributions of team members from Berkeley Lab, Kitt Peak National Observatory, the UC Berkeley Space Sciences Laboratory, and the entire DESI collaboration. As DESI prepares for its survey, we have high hopes for the discoveries that its science team will surely make.

\section{ACKNOWLEDGEMENTS}

This research is supported by the Director, Office of Science, Office of High Energy Physics of the U.S. Department of Energy under Contract No. DE-AC02-05CH1123, and by the National Energy Research Scientific Computing Center, a DOE Office of Science User Facility under the same contract; additional support for DESI is provided by the U.S. National Science Foundation, Division of Astronomical Sciences under Contract No. AST-0950945 to the NSF's National OpticalInfrared Astronomy Research Laboratory; the Science and Technologies Facilities Council of the United Kingdom; the Gordon and Betty Moore Foundation; the Heising-Simons Foundation; the French Alternative Energies and Atomic Energy Commission (CEA); the National Council of Science and Technology of Mexico; the Ministry of Economy of Spain, and by the DESI Member Institutions. The authors are honored to be permitted to conduct astronomical research on Iolkam Du'ag (Kitt Peak), a mountain with particular significance to the Tohono O'odham Nation. 


\section{REFERENCES}

[1] Levi, M., Bebek, C., Beers, T., Blum, R., Cahn, R., Eisenstein, D., Flaugher, B., Honscheid, K., Kron, R., Lahav, O., McDonald, P., Roe, N., Schlegel, D., and representing the DESI collaboration, "The DESI Experiment, a whitepaper for Snowmass 2013," arXiv e-prints , arXiv:1308.0847 (Aug. 2013).

[2] Michael Schubnell, Jon Ameel, Robert W. Besuner, Irena Gershkovich, Philipp Hoerler, Jean-Paul Kneib, Henry D. Heetderks, Joseph H. Silber, Gregory Tarlé, and Curtis Weaverdyck "The DESI fiber positioner system", Proc. SPIE 9908, Ground-based and Airborne Instrumentation for Astronomy VI, 990892 (9 August 2016)

[3] Allen, L., Sprayberry, D., Evatt, M., Marshall, R., Abareshi, B., Lavoie, T., Joyce, R., Probst, R., Dey, A., Blum, R., Besuner, R., Jelinsky, P., Silber, J., Claybaugh, T., Poppett, C., Doel, P., Brooks, D., Gutierrez, G., Flaugher, B., Gallo, G., Friend, D., Sharples, R., Bramall, D., Honscheid, K., Martini, P., Derwent, M., and O’Brien, T., "DESI installation: preparations and progress (Conference Presentation)," in [Groundbased and Airborne Telescopes VII], Marshall, H. K. and Spyromilio, J., eds., 10700, International Society for Optics and Photonics, SPIE (2018).

[4] Yutong Duan, Joseph H. Silber, Todd M. Claybaugh, Steven P. Ahlen, David Brooks, and Gregory Tarlé "Focal plate structure alignment of the Dark Energy Spectroscopic Instrument," Journal of Astronomical Telescopes, Instruments, and Systems 5(1), 014003 (25 January 2019) 